\title{
Estrategias metacognitivas y comprensión lectora en estudiantes universitarios
}

\author{
Noel Alcas Zapata ${ }^{1 a}$, Mitchell Alberto Alarcón Diaz², Henry Hugo Alarcón Diaz ${ }^{3}$, Rosa Gonzáles \\ LLontop ${ }^{4}$, Antonio Rodríguez Fuentes ${ }^{5}$
}

Universidad de San Martín de Porres, Lima, Perú ${ }^{1}$

Universidad Nacional de Educación, Lima, Perú ${ }^{23}$

Universidad Nacional Pedro Ruiz Gallo, Lambayeque, Perú ${ }^{4}$

Universidad de Granada, Granada, España ${ }^{5}$

Orcid ID: https://orcid.org/0000-0002-2505-6778 1

Orcid ID: https://orcid.org/0000-0003-0027-5701 2

Orcid ID: https://orcid.org/0000-0003-1588-4390 3

Orcid ID: https://orcid.org/0000-0002-7516-8022

Orcid ID: https://orcid.org/0000-0002-8036-9902

Recibido: 04 mayo de 2018

Aceptado: 17 de noviembre de 2018

\section{Resumen}

La investigación tuvo como objetivo determinar la influencia de las estrategias metacognitivas en la comprensión lectora de estudiantes de una universidad de Lima (Perú). Metodológicamente, se empleó el método hipotético deductivo, con un enfoque cuantitativo y diseño cuasiexperimental. La muestra estuvo conformada por 62 estudiantes pertenecientes a la especialidad de educación. Los resultados descriptivos e inferenciales permitieron demostrar la influencia de las estrategias de metacognitivas en la comprensión lectora de los participantes. En todos los casos analizados se alcanzó el logro previsto evidenciándose así la efectividad de la intervención realizada.

Palabras clave: metacomprensión, estrategias metacognitivas, comprensión lectora, control de la comprensión.

\section{Metacognitive strategies and reading comprehension in university students}

\author{
Abstract

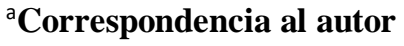 \\ E-mail: nalcas@ucv.edu.pe
}


The objective of this research study was to determine the influence of metacognition on the reading comprehension of students of a university in Lima (Peru). Methodologically, the hypothetical deductive method was used, with a quantitative approach and quasi-experimental design. The sample consisted of 62 students belonging to the specialty of education. The descriptive and inferential results demonstrated the influence of metacognition on the reading comprehension of the participating subjects. In all cases, the expected outcome was achieved, evidencing the effectiveness of the intervention carried out.

Keywords: meta-comprehension, metacognitive strategies, reading comprehension, comprehension control.

\section{Introducción}

\section{Estrategias metacognitivas}

Flavell citado en Medel, Vilanova, Biggio, García, \& Martín (2017, p. 12) refiere que cuando se habla de estrategias metacognitivas se está haciendo referencia al proceso y lo que obtenemos a partir de lo que hacemos para controlar el propio aprendizaje. Así también Flavell sostiene que la metacognición se convierte en un espacio en el cual se organiza y comprende la información provocando el conocimiento y la regulación del mismo. Quizá una pregunta adicional sería cuáles son los procesos que los estudiantes deben incorporar para el correcto encaminando de su aprendizaje (Reif, citado en Saiz-manzanares \& Queiruga, 2017). La incorporación de estos procesos consolidaría un proceso exitoso de aprendizaje. Un ejemplo claro de ello es que la autorregulación del aprendizaje es considerada como un predictor del rendimiento académico (Boekaerts, Pintrich \& Zeidner, 2005; Hoyle, 2013; Lennon, 2010; Vohs \& Baumeister, 2011; Zimmerman, 1989). La necesidad de contar con estudiantes cada vez más autónomos en su aprendizaje, entendiéndose esto último como la capacidad de tomar decisiones y la posibilidad de actuar, motiva a que se siga profundizando teóricamente en el espacio metacognitivo. Estudios relacionados con el tema afirman que aquellos estudiantes que logran mejores resultados presentan un mejor conocimiento metacognitivo (Valenzuela, 2018).

Por su parte, Botero, Alarcón, Palomino, Jiménez y Urrego (2017) afirman que comprender la metacognición es un reto, ya que se está frente a un nuevo paradigma que ha sensibilizado a la comunidad educativa respecto a la comprensión de los procesos cognitivos. El abordaje investigativo de la metacognición ha superado las expectativas en los últimos años, las investigaciones refieren que la comprensión y encaminamiento de los procesos metacognitivos conducen a la automatización y reflexión del propio aprender. 
Flavell asume que, conceptualmente, la metacognición es la forma particular en la que cada individuo emplea estrategias para consolidar su aprendizaje. También añade que la metacognición es el conocimiento de nuestra cognición y de cómo los procesos mentales se organizan para lograr un objetivo académico. Con relación a las fases de la metacognición se afirma que se sintetizan en: la planificación, el monitoreo y la evaluación. La puesta en práctica de estas fases conduciría al aumento de la autorreflexión y autoconciencia (Valenzuela, 2018). Así como lo proponen Tolchinsky (2000), Aparicio (2009) y Tyner (2008), el estudiante universitario iniciante ingresa a una nueva cultura formativa donde las competencias propuestas por las diferentes asignaturas conllevan a la práctica comunicativa en sus diversas dimensiones.

\section{Comprensión lectora}

La necesidad de reflexionar acerca de los niveles de comprensión lectora de los estudiantes universitarios pone sobre la mesa de debate un amplio espectro de dificultades y oportunidades, puesto que el correcto desarrollo del hábito lector aportaría al desarrollo de juicio reflexivo, toma de decisiones, etc., lo que repercutiría en los diversos ambientes académicos donde el estudiante se desenvuelva (Vidal-Moscoso, Manriquez-López, Vidal-Moscoso, \& Manriquez-López, 2016). Como afirman Guzmán y Sánchez (2006), la lectura posibilita el pensamiento crítico, el cual se encuentra íntimamente relacionado con el juicio reflexivo, el pensamiento e inferencias lógicas, la resolución de problemas y la toma de decisiones, tras argumentos coherentes y válidos que aumentan la posibilidad de alcanzar un resultado deseable en cualquier actividad que se realice.

Jiménez, Baridon y Manzanal (2016) refieren que el control de la comprensión lectora permite al lector tener un conocimiento declarativo y procedimental de las acciones que lleva a cabo mientras lee. Su importancia reside en la influencia que ejerce en el éxito o fracaso a la hora de abordar una tarea donde interviene una actividad relacionada con la lectura (Mazzitelli, Maturano y Macías, 2013).

Flower \& Hayes (1981) refieren que tanto leer como escribir son procesos complejos, de ahí la frecuencia de aparición de dificultades (Zuppardo, Rodríguez y Serrano, 2017; Rodríguez, 2017a; 2017b; 2017c; 2018). Los son porque en ellos subyacen determinados subprocesos indispensables para contextualizar el contenido, el propósito y estructura del texto. Griffith y Ruan (2005) mencionan que los individuos emplean la metacognición para validar los procesos puestos en marcha y que tienen como objetivo comprender lo que se lee. 
A partir de lo expuesto por Kinstch (1998), en la evaluación de la comprensión de la lectura se pueden incluir una serie de actividades pos lectura, entendiendo que, en el proceso, el estudiante identificó lo más importante de lo leído jerarquizando la información. En estas actividades predomina la implicación cognitiva y metacognitiva, por cuanto la comunicación escrita una serie de fases, la mayoría de ellas mentales y superiores, de ahí la emergencia de modelos descriptivos y explicativos cognitivos (Rodríguez, 2017a; 2017b; 2017c; 2018).

De esta forma, el objetivo de la investigación fue determinar la influencia de las estrategias metacognitivas en los diferentes niveles de la comprensión lectora. Así, se pretende contribuir a la riqueza teórica existente en ambas variables. En efecto, una de las fases de la comrpensión lectora ha sido denominada metacomprensión lectora. Uno de los propósitos de la universidad es hacer del estudiante un gestor de su evolución cognitiva y con la posibilidad de que reinvente sus procesos de aprendizaje. La puesta en práctica de estudios experimentales favorecerían la mejora del rendimiento académico del estudiantado a partir del fortalecimiento de los procesos de autoregulacion (Botero, Alarcón, Palomino, Jiménez y Urrego, 2017), en este caso lectora.

\section{Metodología}

La investigación corresponde a un enfoque cuantitativo. Acerca de la razón de ser de este enfoque, Hernández, Fernández y Baptista (2014) señalan que éste se caracteriza por ser secuencial, riguroso en su ordenamiento, preestablecido teóricamente y probatorio en la medida que mediante la estadística se intentan probar las hipótesis de investigación. La experimentación fue la característica del trabajo de campo, por tanto, responde a un diseño cuasi experimental.

La población estuvo compuesta por los estudiantes de la Facultad de Educación de una universidad de Lima. La muestra la conformaron 62 sujetos. Al iniciar la investigación se realizó una prueba piloto, que resulto tener una alta confiabilidad. El tratamiento estadístico, descriptivo e inferencial, mediante un pretest y postest, es el más pertinente para valorar la influencia de la metacognición en la comprensión lectora. La intervención pedagógica o tratamiento implicó el desarrollo de actividades apoyadas por separatas, lecturas, trabajos individuales y colaborativos en algunos casos apoyados por recursos multimedia. El instrumento para la medición de la variable dependiente fue elaborado por los autores, y a través de él se ha recopilado los datos de la investigación. 
Respecto del análisis de los datos obtenidos, además del recuento de puntuaciones obtenidas y las frecuencias porcentuales de lectores en cada una de las categorías preasignadas (inicial, logro en proceso y logro previsto), se ha empleado para verificar si las diferencias mostrados por las frecuencias y porcentajes anteriores resultan significativas la prueba de contraste no paramétrica de U de Mann-Whitney, debido a las características de la muestra de estudio y al tipo de muestreo empleado.

\section{Resultados}

La tabla 1 muestra los resultados de la evaluación de la comprensión lectora en los grupos de control y experimental. En referencia al pretest, el 71,9\% de los estudiantes del grupo de control está en el nivel básico de "inicio" frente al 63,3\% del grupo experimental que también está en este mismo nivel. Asimismo, en relación al postest, se observa que 81,3\% del grupo de control está en el nivel medio de "logro en proceso", frente al 100\% del grupo experimental que se ubican en el nivel superior de "logro previsto".

Tabla 1

Tabla cruzada entre la comprensión lectora y el test aplicado a los dos grupos de investigación

\begin{tabular}{|c|c|c|c|c|c|c|c|c|}
\hline & & & & Test & & & & Total \\
\hline & & & & $\begin{array}{l}\text { Pretest } \\
\text { control }\end{array}$ & $\begin{array}{l}\text { Pretest } \\
\text { experimental }\end{array}$ & $\begin{array}{l}\text { postest } \\
\text { control }\end{array}$ & $\begin{array}{l}\text { postest } \\
\text { experimental }\end{array}$ & \\
\hline Comprensió & Inicio & Recuento & & 23 & 19 & 0 & 0 & 42 \\
\hline n lectora & & $\%$ dentro & de & $71,9 \%$ & $63,3 \%$ & $0,0 \%$ & $0,0 \%$ & $33,9 \%$ \\
\hline & & Test & & & & & & \\
\hline & Proceso & Recuento & & 9 & 11 & 26 & 0 & 46 \\
\hline & & $\%$ dentro & de & $28,1 \%$ & $36,7 \%$ & $81,3 \%$ & $0,0 \%$ & $37,1 \%$ \\
\hline & & Test & & & & & & \\
\hline & Logro & Recuento & & 0 & 0 & 6 & 30 & 36 \\
\hline & previsto & $\%$ dentro & de & $0,0 \%$ & $0,0 \%$ & $18,8 \%$ & $100,0 \%$ & $29,0 \%$ \\
\hline & & Test & & & & & & \\
\hline Total & & Recuento & & 32 & 30 & 32 & 30 & 124 \\
\hline & & $\%$ dentro & de & $100,0 \%$ & $100,0 \%$ & $100,0 \%$ & $100,0 \%$ & 100,0 \\
\hline & & Test & & & & & & $\%$ \\
\hline
\end{tabular}

Fuente propia 
También los resultados mostraron que la evaluación del nivel literal de la comprensión lectora en los grupos de control y experimental. Inicialmente, en relación al pretest, el 53,1\% de los estudiantes del grupo de control está en el nivel de "inicio" frente al 56,7\% del grupo experimental que también está en este mismo nivel. Finalmente, en referencia al postest, se observa que el grupo de control se distribuye en dos mitades: un 50\% está en el nivel medio de "logro en proceso" y otro 50\% está en el nivel superior de "logro previsto", frente al contundente 96,7\% del grupo experimental que está en el nivel superior de "logro previsto".

Otro resultado importante es el correspondiente a la evaluación del nivel organizacional de la comprensión lectora en los grupos de control y experimental. En referencia al pretest, el 71,9\% de los estudiantes del grupo de control está en el nivel de "inicio" frente al 60,0\% del grupo experimental que también está en este mismo nivel. Tras el tratamiento, en relación al postest, se observa que en el grupo de control el 53,1\% alcanza el nivel de "logro en proceso", frente al 96,7\% del grupo experimental que llega hasta el nivel de "logro previsto".

En lo que se refiere a la evaluación del nivel inferencial de la comprensión lectora, tanto en los grupos de control y experimental del pretest, el 50,0\% de los estudiantes se sitúa en el nivel de “inicio" frente al 50,0\% que lo hace en el nivel "logro previsto". Finalmente, en relación al postest, se observa que en el grupo de control solo el 59,4\% alcanza el nivel de "logro previsto", frente al $100 \%$ del grupo experimental.

Los resultados de la evaluación del nivel crítico de la comprensión lectora en los grupos de control y experimental determinan que en el pretest existe coincidencia entre uno y otro: el 53,1\% de los estudiantes del grupo de control está en el nivel de "inicio" frente al similar 53,3\% del grupo experimental que también está en este mismo nivel. Al contrario, en la evaluación postest se observa que en el grupo de control el 78,1\% sube al nivel intermedio de "logro en proceso", frente al 96,7\% del grupo experimental que obtiene el nivel superior de "logro previsto".

Un aspecto muy importante en este estudio es el referido a la prueba de normalidad, esta se aplicó con el propósito de establecer si la distribución de los datos de la variable en estudio, se aproximan o no a una distribución normal. Se observaron que los datos que corresponden a la comprensión lectora y sus correspondientes niveles, la significancia es igual a $0,000<0,05$, lo cual implica que los datos de la variable comprensión lectora no se aproximan a la distribución normal. Por consiguiente, el estadístico seleccionado para la prueba de hipótesis es el test de MannWhitney. 
La tabla 2 muestra la prueba de rangos, donde se observa que existe diferencia significativa en la comprensión lectora entre los dos grupos: control y experimental, lo cual se evidencia con el valor de $Z_{\text {calculado }}=-6,793<Z_{\text {critico }}=-1,96$ y el $p=0,000$ menor a $\alpha=0,05$. Por lo tanto, cabe aseverar que las estrategias metacognitivas influyen en el nivel de comprensión lectora de los estudiantes de una universidad de Lima.

Tabla 2

Rangos y nivel de significación del nivel de comprensión lectora de los estudiantes de una universidad de Lima

\begin{tabular}{|c|c|c|c|c|c|}
\hline & Test & $\mathrm{N}$ & $\begin{array}{l}\text { Rango } \\
\text { promedio }\end{array}$ & $\begin{array}{ll}\text { Suma de } \\
\text { rangos }\end{array}$ & $\begin{array}{l}\text { Test U } \\
\text { Mann-Whitney }\end{array}$ \\
\hline \multirow{3}{*}{$\begin{array}{l}\text { Comprensión } \\
\text { lectora }\end{array}$} & Postest control & 32 & 16,69 & 534,00 & $\mathrm{U}=6,000$ \\
\hline & Postest experimental & 30 & 47,30 & 1419,00 & $Z=-6,793$ \\
\hline & Total & 62 & & & Sig. asintót $=0,000$ \\
\hline
\end{tabular}

Fuente propia

En la tabla 3, los resultados específicos a través de la prueba de rangos, demostraron que existe diferencia significativa en el nivel literal de la comprensión lectora entre los dos grupos: control y experimental, lo cual se evidencia con el valor de $Z_{\text {calculado }}=-5,553<Z_{\text {critico }}=-1,96$ y el $\mathrm{p}=0,000$ menor a $\alpha=0,05$. Por lo tanto, se confirma que las estrategias metacognitivas influyen en el nivel literal de comprensión lectora de los estudiantes de una universidad de Lima.

También se encontró que existen diferencias significativas en el nivel organizacional, el nivel inferencial y el nivel crítico de la comprensión lectora entre los dos grupos: control y experimental: la prueba de rangos a través de los valores $Z_{\text {calculado }}=-5,757<Z_{\text {critico }}=-1,96 ; Z_{\text {calculado }}$ $=-4,196<Z_{\text {critico }}=-1,96 ; Z_{\text {calculado }}=-6,401<Z_{\text {critico }}=-1,96$, y en todos los casos menores a $\alpha=$ 0,05 , respectivamente así lo demostraron.

Tabla 3

Rangos y significación del nivel literal de la comprensión lectora de los estudiantes de una universidad de Lima 


\begin{tabular}{|c|c|c|c|c|c|}
\hline \multicolumn{6}{|l|}{ Rangos } \\
\hline & Test & $\mathrm{N}$ & $\begin{array}{l}\text { Rango } \\
\text { promedio }\end{array}$ & $\begin{array}{l}\text { Suma de } \\
\text { rangos }\end{array}$ & 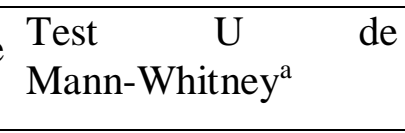 \\
\hline \multirow[t]{3}{*}{ Nivel literal } & Postest control & 32 & 19,38 & 620,00 & $\mathrm{U}=92,000$ \\
\hline & Postest experimental & 30 & 44,43 & 1333,00 & $Z=-5,553$ \\
\hline & Total & 62 & & & Sig. asintót $=0,000$ \\
\hline
\end{tabular}

Fuenre propia

\section{Discusión}

En relación con los resultados de la evaluación de la comprensión, se halló que el grupo control alcanzó, mayoritariamente, un $81.3 \%$, la categoría intermedia de "logro en proceso", frente al 100\% del grupo experimental que alcanzó la máxima categoría de el "logro previsto". Se encontró también que el 96,7\% del grupo experimental alcanzó el "logro previsto" de nivel literal de comprensión; el 96,7\% de nivel organizacional; el 100\% de nivel inferencial, y finalmente el 96,7\% de "logro previsto" de nivel crítico.

Estos hallazgos reafirman la importancia de desarrollar en los estudiantes los procesos que encaminen a mejorar su accionar académico en favor de un mejor aprendizaje (Mato-Vásquez, Espiñeira \& López-Chao, 2017). También se resalta la necesidad de, no solamente conocer cómo mejorar el aprendizaje de los estudiantes, sino también la necesidad de cómo procedimentalmente se podría mejorar el desempeño académico (Cueli, González, Rodríguez, Núñez y González, 2018).

Los resultados inferenciales muestran que las estrategias metacognitivas influyen en el nivel de comprensión lectora, literal, organizacional, inferencial y crítico de la comprensión lectora. Estos hallazgos coinciden con Muñoz y Ocaña (2017) quienes afirman que el desarrollo de talleres promueve la activación de procesos sobre lo que se reflexiona acerca del propio actuar, el despertar de la agudeza y la habilidad para efectivizar el proceso lector.

\section{Referencias}

Botero, A., Alarcón, D., Palomino, D., Jiménez, Á., \& Urrego, Á. (2017). Pensamiento crítico, metacognición y aspectos motivacionales: una educación de calidad. Poiésis, 1(33), 85. https://doi.org/10.21501/16920945.2499

Cueli, M., González, P., Rodríguez, C., Núñez, J., \& González, J. (2018). Efecto de una herramienta hipermedia sobre las variables afectivo-motivacionales relacionadas con las 
matemáticas. Revista $\quad$ Educación $\quad X X I . \quad 2(1), \quad 375-394$. https//doi.org/10.5944/educxx1.20211

Diaz, J. S. S., \& Pérez Zapata, D. I. (2017). Concepción de aprendizaje en estudiantes universitarios de la carrera de psicología del extremo norte de Chile. Universitas Psychologica, 16(2). https://doi.org/10.11144/Javeriana.upsy16-2.caeu

Griffith, P.\& Ruan, J. (2005). What is metacognition and what should be its role in literacy instruction? In S. E. Israel, C. C. Block, K. L. Bauserman, \& K. Kinnucan-Welsch (Eds.), Metacognition in literacy learning: Theory, assessment, instruction, and professional development (pp. 3-18). Mahwah, NJ: Lawrence Erlbaum Associates.

Hernández, A., \& Camargo, Á. (2017). Autorregulación del aprendizaje en la educación superior en Iberoamérica: una revisión sistemática. Revista Latinoamericana de Psicología, 49(2), 146-160. https://doi.org/10.1016/j.rlp.2017.01.001

Hernández, R. Fernández C., \& Baptista, M. (2014). Metodología de la investigación. México: McGraw-Hill/Interamericana.

Izquierdo-Magaldi, B., Renés-Arellano, P., \& Gómez-Cash, O. (2016). Estrategias metacognitivas y recursos tecnológicos utilizados por estudiantes universitarios de español como segunda lengua. Ocnos: Revista de Estudios Sobre Lectura, 15(1), 149. https://doi.org/10.18239/ocnos_2016.15.1.958

Jimenez, L., Baridon, D., \& Manzanal, A. (2016). Análisis del control de la comprensión lectora en textos científicos en alumnos de Secundaria Obligatoria y Bachillerato. Revista $\begin{array}{lllll}\text { Complutense } & \text { de } & \text { Educación. } & 27 & \text { (307), }\end{array}$ 307. http://dx.doi.org/10.5209/rev_RCED.2016.v27.n1.45749

Mato-Vázquez, D., Espiñeira, E., \& López-Chao, V. (2017). Impacto del uso de estrategias metacognitivas en la enseñanza de las matemáticas. Perfiles educativos, 39(158), 91-111. Recuperado de 2018, de http://www.scielo.org.mx/scielo.php?script=sci_arttext\&pid=S0185$26982017000400091 \& \operatorname{lng}=\mathrm{es} \& \operatorname{lng}=$.

Mazzitelli, C., Maturano, C., \& Macias, A. (2013). Dificultades estratégicas en la comprensión lectora de estudiantes de Ciencias Naturales. Revista Electrónica de Investigación en Educación en Ciencias. 8(2), 33-48. Recuperado de http://www.redalyc.org/comocitar.oa?id=273330004004

Medel, G. A., Vilanova, S. L., Biggio, C., García, M. B., \& Martín, S. S. (2017). Estrategias metacognitivas y concepciones sobre el aprendizaje en la formación inicial de profesores universitarios del área de ciencias exactas y naturales. Informes Psicológicos, 17(1), 35-51. https://doi.org/10.18566/infpsic.v17n1a02

Muñoz-Muñoz, Á., \& Ocaña, M. (2017). Uso de estrategias metacognitivas para la comprensión textual. Cuadernos de Lingüística Hispánica. (29), 223-244. doi: http://dx.doi.org/10.19053/0121053X.n29.2017.586 
Olivo, J. (2017). Caracterización de estudiantes exitosos: Una aproximación al aprendizaje de las Ciencias Naturales. CPU-e Revista de Investigación Educativa. 25, 114 - 143. Recuperado de http://www.redalyc.org/articulo.oa?id=283152311006

Rodríguez, A. (2006). Programas para desarrollar el lenguaje escrito, en J.L. Gallego (dir.), Enciclopedia temática de Logopedia. Málaga: Aljibe.

Rodríguez, A. (2017a). Evaluación de dificultades lectoras escolares. En M. El Homrani, F. Peñafiel y A. Hernández (cords.), Entornos y estrategias educativas para la inclusión social. Granada: Comares.

Rodríguez, A. (2017b). Simposio sobre evaluación de dificultades lectoescritoras. En II Congreso Internacional SEI 2017: Sociedad, Educación e Inclusión. Universidad de Granda y Asociación UGREDUSO (Universidad de GRAnada. EDUcación Social). Granada (España). 26 de Abril de 20147.

Rodríguez, A. (2017c). Modelos cognitivos descriptivos de Lectura y Escritura e intervención didáctica. Conferencia en el Instituto Panameño de Habilitación Especial. Ciudad de Panamá (Panamá), 20-24 de Marzo de 2017.

Rodríguez, A. (2018). Dificultades en la lectoescritura. Conferencia en la Universidad Central "Marta Abreu" de las Villas (Cuba). Santa Clara (Cuba), 5 de Febrero de 2018.

Saiz-manzanares, M., \& Queiruga, M. A. (2017). Evaluación de estrategias metacognitivas: aplicación de métodos online. Revista de Psicología y Educación - Journal of Psychology and Education, 13(February 2018), 45. https://doi.org/10.23923/rpye2018.01.156

Valenzuela, Á. A. (2018). La metacognición en los procesos de lectura y escritura académica: ¿qué nos dice la literatura? Lenguaje, 46(1), 69. https://doi.org/10.25100/lenguaje.v46i1.6197

Vidal-Moscoso, D., Manriquez-López, L., Vidal-Moscoso, D., \& Manriquez-López, L. (2016). El docente como mediador de la comprensión lectora en universitarios. Revista de La Educación Superior, 45(177), 95-118. https://doi.org/10.1016/j.resu.2016.01.009

Zuppardo, L., Rodríguez, A., \& Serrano, F. (2017). Modelo piloto de estudio del tratamiento rehabilitador de autoestima y comportamiento en adolescentes con dislexia y disortografía mediante su mejora lectoescritora. Propósitos y Representaciones, 5(2), 359-400. doi: http://dx.doi.org/10.20511/pyr2017.v5n2.174 